\title{
Reduced electronic correlation effects in half substituted $\mathrm{Ba}\left(\mathrm{Fe}_{1-\mathrm{x}} \mathrm{Co}_{\mathrm{x}}\right)_{\mathbf{2}} \mathrm{As}_{\mathbf{2}}$
}

\author{
Z.-H. Liu, ${ }^{1,2, a)}$ A. N. Yaresko, ${ }^{3}$ Y. Li, ${ }^{4}$ D. V. Evtushinsky, ${ }^{1}$ P.-C. Dai, ${ }^{4}$ and S. V. Borisenko ${ }^{1, b)}$ \\ ${ }^{1}$ Institute for Solid State Research, IFW Dresden, D-01171 Dresden, Germany \\ ${ }^{2}$ State Key Laboratory of Functional Materials for Informatics, SIMIT, Chinese Academy of Sciences, \\ Shanghai 200050, China \\ ${ }^{3}$ Max Planck Institute for Solid State Research, D-70569 Stuttgart, Germany \\ ${ }^{4}$ Department of Physics and Astronomy, Rice University, Houston, Texas 77005-1827, USA
}

(Received 11 April 2018; accepted 28 May 2018; published online 8 June 2018)

\begin{abstract}
We report a comprehensive study of the tridimensional nature and orbital character of the lowenergy electronic structure in $50 \%$ Cobalt doped $\mathrm{Ba}\left(\mathrm{Fe}_{1-\mathrm{x}} \mathrm{Co}_{\mathrm{x}}\right)_{2} \mathrm{As}_{2}\left(d^{6.5}\right)$, by using polarizationand photon energy-dependent angle-resolved photoemission spectroscopy. An extra electron-like Fermi surface is observed around the Brillouin zone boundary compared with isoelectronic $\mathrm{K}_{\mathrm{y}} \mathrm{Fe}_{2-\mathrm{x}} \mathrm{Se}_{2}\left(d^{6.5}\right)$. The bands near the Fermi level $\left(\mathrm{E}_{\mathrm{F}}\right)$ are mainly derived from $\mathrm{Fe} / \mathrm{Co} 3 d t_{2 g}$ orbitals, revealing visible dispersions along the $k_{z}$ direction. In combination with the local density approximation and the dynamical mean-field theory calculations, we find that the As $4 p$ bands are non-renormalized and the whole $3 d$ band needs to be renormalized by a "single" factor of $\sim 1.6$, indicating moderate electronic correlation effects. The "single" factor description of the correlation strength among the different $3 d$ orbitals is also in sharp contrast to orbital-dependent correlation effects in $\mathrm{BaFe}_{2} \mathrm{As}_{2}$. Our findings indicate a remarkable reduction of correlation effects with little difference among $3 d$ orbitals in $\mathrm{BaFeCoAs}_{2}$, due to the increased filling of the electronic $3 d$ shell in the presence of significant Hund's coupling. The results support that the electronic correlation effects and multiple orbital physics play an important role in the superconductivity of the 122 system and in other ferropnictides. Published by AIP Publishing. https://doi.org/10.1063/1.5034488
\end{abstract}

By application of either high pressure or chemical substitution, the superconductivity can be induced in ferropnictide, ${ }^{1,2}$ which is a family of superconducting compounds with critical temperatures $\left(T_{c}\right)$ being the second highest to those of the cuprates. In the case of cuprates, the Mott insulating ground state of the parent non-superconducting materials inspired theorists to describe high temperature superconductivity originating from a strongly correlated matter. Heated debates for electronic correlation of iron-based superconductors (IBSs) have been going on. ${ }^{3-5}$ On the one hand, the lowenergy electronic band structure is well described by the mean field theory calculation, ${ }^{6}$ which suggests the property of itinerant electrons. On the other hand, the bandwidth renormalization is needed to capture the essential dispersive features according to the results of the dynamical mean-field theory $(\mathrm{DMFT})^{7,8}$ or the angle-resolved photoemission spectroscopy (ARPES), ${ }^{9-15}$ which indicates non-negligible electronic correlations in IBS.

The band structure of the 122 system is extensively studied by theoretical and experimental methods since doping of both electrons $\left[\mathrm{Ba}\left(\mathrm{Fe}_{1-\mathrm{x}} \mathrm{Co}_{\mathrm{x}}\right)_{2} \mathrm{As}_{2}\right]$ and holes $\left(\mathrm{Ba}_{1-\mathrm{x}} \mathrm{K}_{\mathrm{x}} \mathrm{Fe}_{2} \mathrm{As}_{2}\right)$ can suppress magnetic order and lead to a dome of superconductivity before reaching a more conventional metal or Mott insulator. The dopant not only introduces extra carriers and shifts the chemical potential up or down but also further tunes $3 d$ shell away from or close to the half filling, which signifies a Mott insulator in strong correlation theory owing to the strong coulomb interaction. A study of hole over-doped $\mathrm{BaFe}_{2} \mathrm{As}_{2}$ evidenced an unusual non-Fermi-liquid behavior with frozen

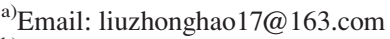

b)Email: s.borysenko@ifw-dresden.de
}

moments in the paramagnetic phase, indicating the strong band renormalization near the Fermi level $\left(\mathrm{E}_{\mathrm{F}}\right){ }^{8}$ Along with the increased filling of the electronic $3 d$ shell and the decreased strength of electronic correlation, the crossover is found toward a Hund's metal state, for which the correlation is more sensitive to $J$ than to $U{ }^{16-19}$ Additionally, one finds that a single global renormalization factor does not suit each band. ${ }^{13-15}$ Hund's rule coupling dominates the orbital-dependent correlation strength in the expected regime, where the $t_{2 g}$ orbitals are more correlated than $e_{g}$ ones; in particular, the $d_{x y}$ orbital is the most correlated of all. ${ }^{5,20}$ These results exhibit that electronic correlation and the multi-orbital physics play an important role in the system.

In this work, we present ARPES results on 50\% Cobalt doped $\mathrm{Ba}\left(\mathrm{Fe}_{1-\mathrm{x}} \mathrm{Co}_{\mathrm{x}}\right)_{2} \mathrm{As}_{2}$ using different polarized photons in a wide energy range. The number of $3 d$ electrons of $\mathrm{BaFeCoAs}_{2}\left(d^{6.5}\right)$ is at the middle of $\mathrm{BaFe}_{2} \mathrm{As}_{2}\left(d^{6}\right)$ and $\mathrm{BaCo}_{2} \mathrm{As}_{2}\left(d^{7}\right)$. We observed one electron-like Fermi surface (FS) at the Brillouin zone (BZ) center, one electron-like FS near the zone boundary, and two large electron-like FSs at the zone corner. They mainly originate from the Fe/Co $3 d t_{2 g}$ orbitals with evident $k_{z}$ dispersions. The chemical potential shifts up about $0.24 \mathrm{eV}$ compared with the parental $\mathrm{BaFe}_{2} \mathrm{As}_{2}$. ${ }^{11,21-23}$ In combination with the local density approximation (LDA) and the DMFT calculations, we find that an overall band renormalization of about 1.6 is needed to capture the essential dispersive features, indicating much weaker correlation effects than in $\mathrm{BaFe}_{2} \mathrm{As}_{2}$ and a relatively strong correlation effect than in $\mathrm{BaCo}_{2} \mathrm{As}_{2}$, for which the renormalization factors of 3 and 1.4 were reported, respectively. ${ }^{11,24}$ Unlike in the regime of hole-doped and electronic under-doped $\mathrm{BaFe}_{2} \mathrm{As}_{2},{ }^{13-15}$ the electronic correlation strengths are little different among $3 d$ 

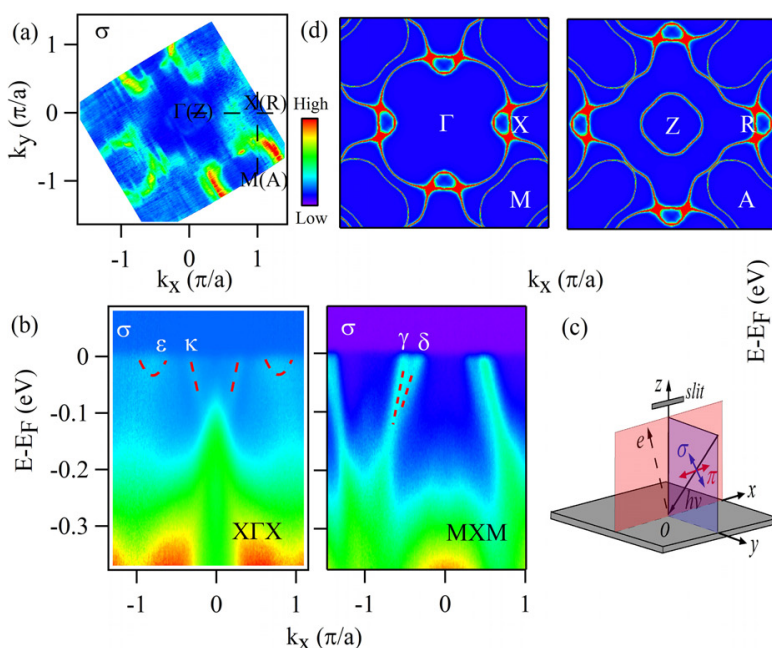

$\mathrm{k}_{\mathrm{X}}(\pi / \mathrm{a})$

(c)
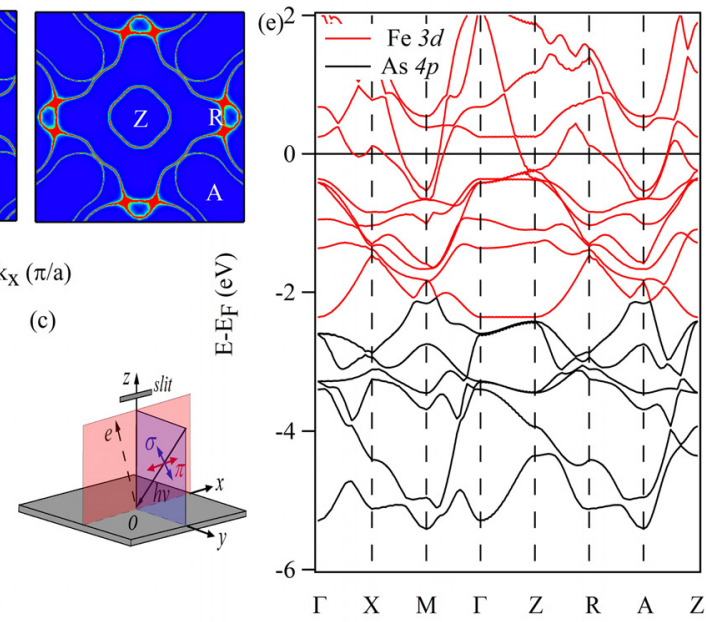

FIG. 1. (a) Integrated intensity plot at $\mathrm{E}_{\mathrm{F}}( \pm 10 \mathrm{meV})$ of $\mathrm{BaFeCoAs}_{2}$ in $\sigma$ geometry using $h \nu=80 \mathrm{eV}$ at T $=1 \mathrm{~K}$. (b) Intensity plots of band dispersion along ХГХ and MXM directions, as indicated in (a). Dashed lines are guides to the eye for the band dispersions. (c) Sketch of the experimental polarization setup. The $\sigma$ and $\pi$ polarizations are for electric fields perpendicular and parallel to the mirror plane (red), respectively. (d) Calculated FSs at ГXM and ZRA planes using the LDA calculation. The calculation details will be stated in the content later. (e) LDA bands along the high symmetry lines in the first BZ. orbitals in $\mathrm{BaFeCoAs}_{2}$. Combined with previous studies, our findings support that Hund's rule coupling plays the crucial role in ferropnictide materials. ${ }^{16,17,20,25,26}$ The strength of electronic correlations is efficiently tuned by changing the filling of the electronic $3 d$ shell.

High quality single crystals of $\mathrm{BaFeCoAs}_{2}\left(\mathrm{~T}_{\mathrm{c}}=0\right)$ were synthesized by the flux method. Samples with the size smaller than $1 \times 1 \mathrm{~mm}^{2}$ were cleaved in situ, yielding a flat mirror like (001) surface. ARPES measurements were performed using synchrotron radiation, 1-cubed ARPES end station at BESSY, within a wide range of photon energies and various polarizations. The optimum energy and momentum resolutions were $\sim 10 \mathrm{meV}$ and $\sim 0.02 \AA^{-1}$, respectively, for the low temperature measurements. During measurements, the pressure was maintained better than $5 \times 10^{-11}$ Torr. From our experimental setup shown in Fig. 1(c), we can observe that the high-symmetry directions and the normal of the sample surface define a mirror plane. The $\pi(\sigma)$ geometry refers to the electric fields of the incident photons within (normal to) the mirror plane, respectively. The even (odd) orbitals with respect to the mirror plane are detected in $\pi(\sigma)$ geometry. Here, we note that our experimental setup for the $\sigma$ geometry includes a polarization component along $z$.

Figure 1(a) shows the measured FS map of $\mathrm{BaFeCoAs}_{2}$ at $1 \mathrm{~K}$ using $80 \mathrm{eV}$ in $\sigma$ geometry. To identify which FSs are represented by intensity spots on the map, we have recorded the energy-momentum distributions of the photocurrents along the XГX (RZR) and MXM (ARA) direction, as shown in Fig. 1(b). There are four electron-like bands near $E_{F}$, i.e., one at the zone center, one near the zone boundary, and two centered around the zone corner, named $\kappa, \varepsilon, \gamma$, and $\sigma$, respectively. In comparison with typical FS topology in IBS, ${ }^{9-14}$ an additional electron-like FS turns up at the zone boundary, which is consistent with calculated FS, as shown in Fig. 1(d). The measured FS at $80 \mathrm{eV}$ is rather similar to the calculated FS at the ГXM plane, indicating that the photon energy of $80 \mathrm{eV}$ closely corresponds to the $\Gamma$ plane along the $k_{z}$ direction. Comparing the FSs at the ГXM plane and the ZRA plane in Fig. 1(d), it can be observed that all of FSs show visible $k_{z}$ dispersion, revealing the 3-dimensional (3D) nature of FSs in $\mathrm{BaFeCoAs}_{2}$. Figure 1(e) shows the LDA calculated bands along the high symmetry lines in the BZ. Indeed, the low-energy electron dynamics in the 122 system is defined by
$3 d$ electrons and the only principal difference is that the half of these $3 d$ electrons in the case of $\mathrm{BaFeCoAs}_{2}$ stems from Co atoms. Apparently, this is not a significant variation from the point of view of the LDA theory. 8,9

To investigate the $k_{z}$ dispersion, we carried out measurements in $\pi$ geometry using photon energies $(h \nu)$ from 28 to $90 \mathrm{eV}$, which covers more than $1.5 \mathrm{BZs}$ along $k_{z}$. Figure 2(a) shows the intensity at $\mathrm{E}_{\mathrm{F}}$ as a function of photon energy and $k_{/ /}$oriented along the $\Gamma \mathrm{X}(\mathrm{ZR})$ direction. This plot implies that the $\kappa$ band has a strong $k_{z}$ dispersion, with a band minimum at $\Gamma(\sim 48,76 \mathrm{eV})$ and a band maximum at $\mathrm{Z}(\sim 36$, $62 \mathrm{eV}$ ). The $k_{z}$ variation of the $\kappa$ band is also well illustrated by the intensity plots along the $Г \mathrm{M}$ and ZA lines, as shown in Figs. 2(b) and 2(c), respectively. Comparing the top of the $\alpha$ band at the $\Gamma$ point and $\mathrm{Z}$ point, one finds an appreciable $k_{z}$ variation of $\alpha$ with an opposite tendency of that in $\kappa$.

Figures 2(c) and 2(d) show the intensity plots of band dispersion along ZA in $\pi$ and $\sigma$ geometries, respectively. The data taken with different geometries allow us to detect the orbital character of the bands. According to the symmetry analysis and by analogy with others IBS, ${ }^{12-15,27-31}$ we identify the orbital character of each band. At the $\mathrm{Z}$ point,
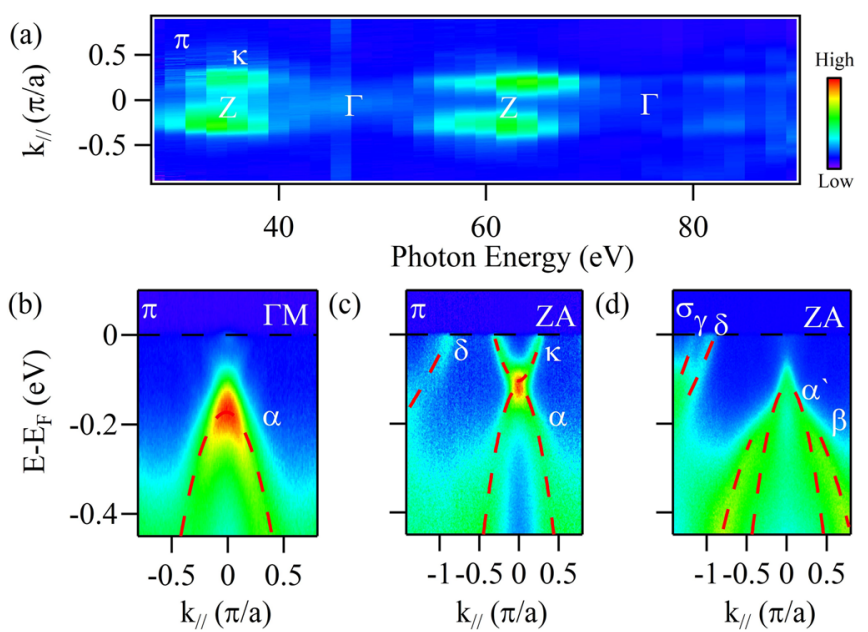

FIG. 2. (a) Intensity plot in the $h \nu$-k// plane of $\mathrm{BaFeCoAs}_{2}$ in $\pi$ geometry $\left( \pm 10 \mathrm{meV}\right.$ integration centered at $\mathrm{E}_{\mathrm{F}}$ ). (b) Intensity plot of band dispersion along $\Gamma \mathrm{M}(48 \mathrm{eV})$ in $\pi$ geometry. (c) and (d) Intensity plots of band dispersion along ZA $(62 \mathrm{eV})$ in $\pi$ and $\sigma$ geometries, respectively. The dashed lines are guides to the eye. 
the $\alpha$ and $\kappa$ bands are enhanced only in $\pi$ geometry. They should be of $d_{x z}$ character. The $\alpha^{\prime}$ and $\beta$ bands are highlighted only in $\sigma$ geometry, and they have $d_{y z}$ and $d_{x y}$ orbital character. At the A point, the $\delta$ band can be seen in both geometries and the $\gamma$ band is only detected in $\sigma$ geometry, suggesting that $\delta$ is of $d_{x z}$ and $d_{y z}$ and $\gamma$ is of $d_{x y}$ orbital character.

After study of the FSs and the band structure with their orbital character by ARPES, we investigated the electronic correlation by comparing the energy bandwidths between experiments and calculations. We performed calculations based on the LDA + DMFT for $\mathrm{BaFeCoAs}_{2}$ with the experimental crystal structural parameters $\mathrm{a}=3.955 \AA$ and $\mathrm{c}=12.813 \AA$. The internal coordinate, $\mathrm{z}_{\mathrm{As}}=0.35405$, was relaxed by energy minimization for the ordered cell. The interaction parameters were chosen as $\mathrm{u}=5.0 \mathrm{eV}$ and $\mathrm{J}=0.9 \mathrm{eV}$. The LDA results were obtained using the PY LMTO computer code. ${ }^{32}$ The DMFT was performed with WIEN2K and the ALPS CTQMC hybridization solver with full charge-selfconsistency.

Figure 3 shows the experimental band dispersions along the high symmetry directions in $\pi$ and $\sigma$ geometries, respectively. LDA calculated bands were appended with red $(3 d$ bands) and black ( $4 p$ bands) curves. The $3 d$ bands near $\mathrm{E}_{\mathrm{F}}$ calculated by LDA need to be narrowed 1.6 times to fit the experimental data and the $4 p$ bands at high bind energy do not need any narrowing. The unnormalized $4 p$ bands are also found in NaFeAs and FeSe compounds. ${ }^{33,34}$ To much accurately survey the renormalization factor of the $3 d$ bands, we presented the second derivative intensity plot along ZRA lines in $\pi$ and $\sigma$ geometries appending renormalized LDA bands by a factor of 1.6, as shown in Fig. 4(a). The data taken in $k_{z}=\pi$ plane with two different geometries allow us to detect all $3 d$ bands near $\mathrm{E}_{\mathrm{F}}$. The $\omega$ band with $d_{z 2}$ character is enhanced by the $z$ component in the $\sigma$ geometry. Indeed, a "single" renormalization factor of 1.6 is only needed to capture the essential dispersive features. Besides, we also employed the DMFT to study the factor of the $3 d$ bands with different orbital characters. The DMFT results appended with 1.6 times narrowed density functional theory (DFT) calculation bands are shown in Fig. 4(b). The orbital dependence renormalization factors in DMFT are 1.588, 1.564, 1.651 , and 1.591 for $x z / y z, x y, x^{2}-y^{2}$, and $z^{2}$, respectively. Apparently, the numbers change just a little bit. Although the FS topology is no change upon the introduction of correlations, there is a significant mismatch of the bands right above $\mathrm{E}_{\mathrm{F}}$ at the $\Gamma$ point [marked by the red circle in Fig.
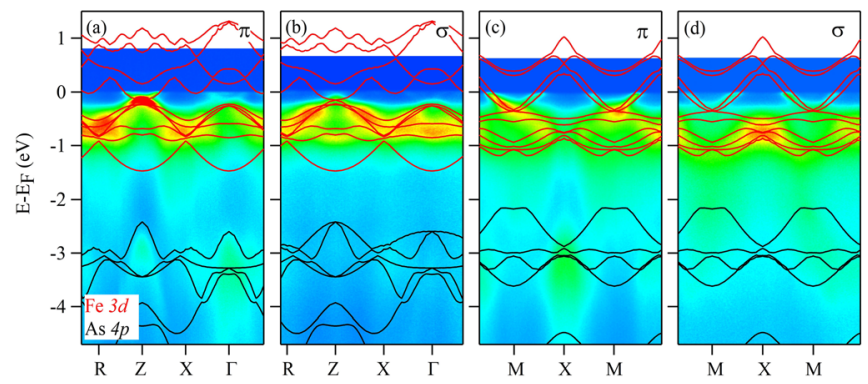

FIG. 3. The experimental band dispersions along the high symmetry directions of $\mathrm{BaFeCoAs}_{2}$ in $\pi$ and $\sigma$ geometries, respectively. LDA calculated bands were appended with red ( $3 d$ bands) and black ( $4 p$ bands) curves. The $3 d$ bands calculated by LDA are narrowed 1.6 times to fit the experimental data and the $4 p$ bands are not narrowed.
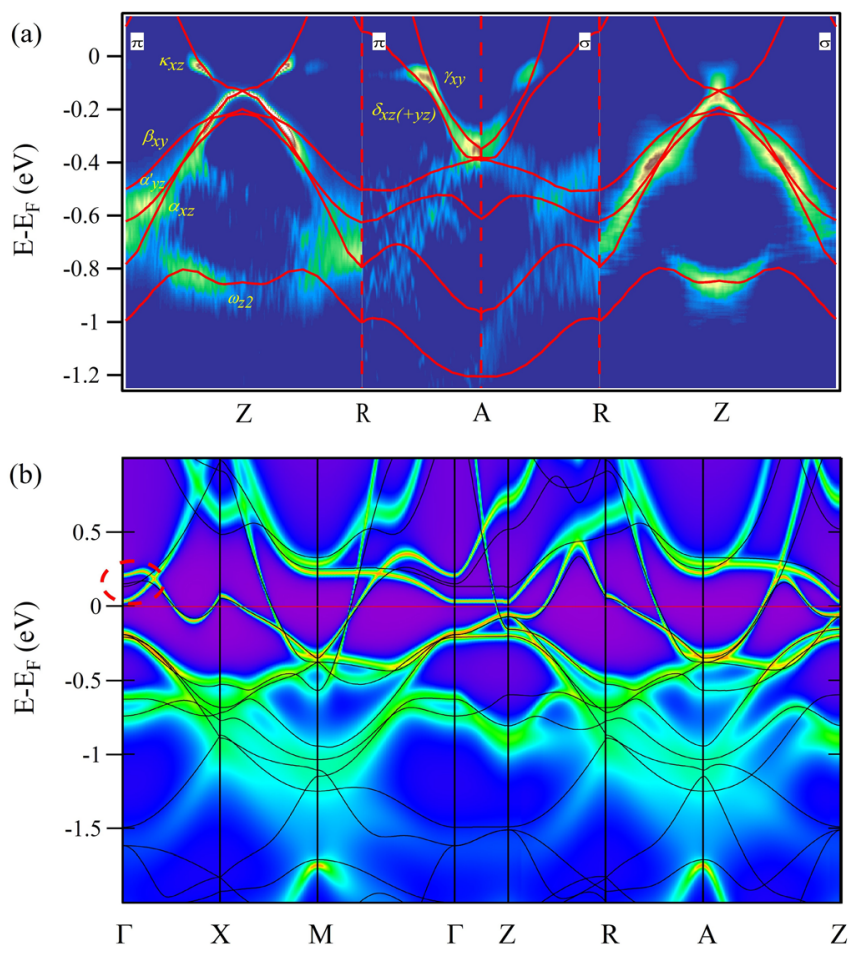

FIG. 4. (a) Second derivative intensity plot along ZRA lines of $\mathrm{BaFeCoAs}_{2}$ in $\pi$ and $\sigma$ geometries, appending renormalized LDA bands by a factor of 1.6. The orbital characters and polarizations are indicated. (b) LDA + DMFT calculations along the high symmetry lines. The bright color lines are calculated by the LDA + DMFT, and the black lines correspond to the DFT calculated bands renormalized by a factor of 1.6.

4(b)], which can be explained in terms of correlation effects and the evidence of inter-band coupling. The downshift band can account for the shadow near $\mathrm{E}_{\mathrm{F}}$ at the $\Gamma$ point, as shown in Figs. 1(a), 2(a), and 2(b).

As demonstrated above, the calculated $3 d$ bands need to be renormalized by a "single" factor of $\sim 1.6$ to well duplicate the experimental data. Our finding suggests that the electronic correlation effects in $\mathrm{BaFeCoAs}_{2}\left(d^{6.5}\right)$ are much weaker than in parental $\mathrm{BaFe}_{2} \mathrm{As}_{2}\left(d^{6}\right)$ and little stronger than in fully electron doped $\mathrm{BaCo}_{2} \mathrm{As}_{2}\left(d^{7}\right)$, for which the renormalization factors of 3 and 1.4 were reported. ${ }^{11,24}$ Additional, the correlation strengths were little different among orbitals, unlike in the regime of electron underdoped and hole-doped $\mathrm{BaFe}_{2} \mathrm{As}_{2}$, where the $d_{x y}$ orbital is obviously prominent. ${ }^{13-15}$ The overall view of the 122 system and the electronic correlation strengths are tuned by the filling of electrons in the $3 d$ shell. In the superconducting region, it is dominated by Hund's rule coupling, which in turn decouples the charge excitations in different orbitals, so that the system reveals orbital-dependent electronic correlation effects. ${ }^{5}$ Along with more electrons filling the $3 d$ shell, the system comes into moderately correlated Fermi liquid, as in $\mathrm{BaFeCoAs}_{2}$. In this process, the electronic correlation strength of the $t_{2 g}$ orbital, $d_{x y}$ in particular, evidently reduces and follows the global tendency. In consideration of the correlation effects helping to get high- $\mathrm{T}_{\mathrm{c}}$ superconductors, the hole doped $\mathrm{Ba}_{1-\mathrm{x}} \mathrm{K}_{\mathrm{x}} \mathrm{Fe}_{2} \mathrm{As}_{2}$ should hold a much larger superconducting region and higher $\mathrm{Tc}$ than electron doped $\mathrm{Ba}\left(\mathrm{Fe}_{1-\mathrm{x}} \mathrm{Co}_{\mathrm{x}}\right)_{2} \mathrm{As}_{2}$.

In addition, 1.6 times narrowing of the low-energy dispersions on $\mathrm{BaFeCoAs}_{2}$ is also less than in an isoelectronic 
$\mathrm{K}_{\mathrm{y}} \mathrm{Fe}_{2-\mathrm{x}} \mathrm{Se}_{2}\left(d^{6.5}\right)$, for which 2.5 times narrowing was reported. ${ }^{11}$ In $\mathrm{K}_{\mathrm{y}} \mathrm{Fe}_{2-\mathrm{x}} \mathrm{Se}_{2}$, local antiferromagnetic exchange interaction was proposed as a serious candidate for the pairing mechanism. ${ }^{35}$ We consider two differences of the two materials to explain that why $\mathrm{K}_{\mathrm{y}} \mathrm{Fe}_{2-\mathrm{x}} \mathrm{Se}_{2}$ is a superconductor and the isoelectronic $\mathrm{BaFeCoAs}_{2}$ is not. From the view of the real space, the antiferromagnetic exchange between $\mathrm{Fe}-\mathrm{Fe}$ atoms can be destroyed by the heavy Co doping. While in the respect of the momentum space, the additional electron-like FS at the zone boundary increases the density of states near $\mathrm{E}_{\mathrm{F}}$ and causes the itinerant behavior of the $3 d$ electrons. In electron fully doped $\mathrm{BaCo}_{2} \mathrm{As}_{2}$, the electronic correlation is much less important than in the ferropnictide. ${ }^{23}$ The itinerant electron behavior is suggested in $\mathrm{BaCo}_{2} \mathrm{As}_{2}$ by observation of ferromagnetic characters in resistivity measurements ${ }^{36}$ and nuclear magnetic resonance (NMR) experiments. ${ }^{37}$

In summary, we study the FSs and the low-energy band structure on $\mathrm{BaFeCoAs}_{2}$ by using the ARPES and the LDA + DMFT calculations. The system shows moderately correlated Fermi liquid behavior. Combined with previous studies, our findings indicate strong doping- and orbitaldependent correlation effects in 122 compounds.

We thank Professor Roser Valentí and Dr. Steffen Backes for providing the DFT + DFMT results. This work was supported by the German Science Foundation under Grant No. BO 1912/7-1, the National Natural Science Foundation of China (Nos. 11227902 and 11704394), and the Shanghai Sailing Program (17YF1422900). The single crystal growth work at Rice was supported by the U.S. DOE, BES under Contract No. DE-SC0012311 (P.D.). A part of the materials work at Rice was supported by the Robert A. Welch Foundation under Grant No. C-1839 (P.D.).

${ }^{1}$ Y. Kamihara, T. Watanabe, M. Hirano, and H. Hosono, J. Am. Chem. Soc. 130, 3296 (2008).

${ }^{2}$ Z. A. Ren, G. C. Che, X. L. Dong, J. Yang, W. Lu, W. Yi, X. L. Shen, Z. C. Li, L. L. Sun, F. Zhou, and Z. X. Zhao, Europhys. Lett. 83, 17002 (2008).

${ }^{3}$ M. M. Qazilbash, J. J. Hamlin, R. E. Baumbach, L. J. Zhang, D. J. Singh, M. B. Maple, and D. N. Basov, Nat. Phys. 5, 647 (2009).

${ }^{4}$ W. L. Wang, A. P. Sorini, C. C. Chen, B. Moritz, W. S. Lee, F. Vernay, P. Olalde-Velasco, J. D. Denlinger, B. Delley, J. H. Chu, J. G. Analytis, I. R. Fisher, Z. A. Ren, J. Yang, W. Lu, Z. X. Zhao, J. Van den Brink, Z. Hussain, Z. X. Shen, and T. P. Devereaux, Phys. Rev. B 80, 014508 (2009).

${ }^{5}$ L. de' Medici, G. Giovannetti, and M. Capone, Phys. Rev. Lett. 112, 177001 (2014).

${ }^{6}$ D. J. Singh and M. H. Du, Phys. Rev. Lett. 100, 237003 (2008).

${ }^{7}$ Z. P. Yin, K. Haule, and G. Kotilar, Nat. Mater. 10, 932 (2011).

${ }^{8}$ P. Werner, M. Casula, T. Miyake, F. Aryasetiawan, A. J. Millis, and S. Biermann, Nat. Phys. 8, 331 (2012).

${ }^{9}$ Z. H. Liu, A. N. Yaresko, Y. Li, P. C. Dai, H. Zhang, B. Buchner, C. T. Lin, and S. V. Borisenko, J. Phys.: Condens. Matter 29, 085503 (2017).

${ }^{10}$ S. V. Borisenko, V. B. Zabolotnyy, D. V. Evtushinsky, T. K. Kim, I. V. Morozov, A. N. Yaresko, A. A. Kordyuk, G. Behr, A. Vasiliev, R. Follath, and B. Büchner, Phys. Rev. Lett. 105, 067002 (2010).

${ }^{11}$ P. Richard, K. Nakayama, T. Sato, M. Neupane, Y. M. Xu, J. H. Bowen, G. F. Chen, J. L. Luo, N. L. Wang, X. Dai, Z. Fang, H. Ding, and T. Takahashi, Phys. Rev. Lett. 104, 137001 (2010).
${ }^{12}$ Z. H. Liu, P. Richard, N. Xu, G. Xu, Y. Li, X. C. Fang, L. L. Jia, G. F. Chen, D. M. Wang, J. B. He, T. Qian, J. P. Hu, H. Ding, and S. C. Wang, Phys. Rev. Lett. 109, 037003 (2012).

${ }^{13}$ T. Yoshida, S. Ideta, I. Nishi, A. Fujimori, M. Yi, R. G. Moore, S. Mo, D. H. Lu, Z. X. Shen, Z. Hussain, K. Kihou, P. M. Shirage, H. Kito, C. Lee, A. Iyo, H. Eisaki, and H. Harima, Front. Phys. 2, 17 (2014).

${ }^{14}$ V. Brouet, M. F. Jensen, A. Nicolaou, A. Taleb-Lbrahimi, P. L. Fevre, F. Bertran, A. Forget, and D. Colson, e-print arXiv:1105.5604.

${ }^{15}$ H. Ding, K. Nakayama, P. Richard, S. Souma, T. Sato, T. Takahashi, M. Neupane, Y. M. Xu, Z. H. Pan, A. V. Fedorov, Z. Wang, X. Dai, Z. Fang, G. F. Chen, J. L. Luo, and N. L. Wang, J. Phys.: Condens. Matter 23, 135701 (2011).

${ }^{16} \mathrm{H}$. Ishida and A. Liebsch, Phys. Rev. B 81, 054513 (2010).

${ }^{17}$ R. Yu and Q. Si, Phys. Rev. B 86, 085104 (2012).

${ }^{18}$ N. Lanatà, H. U. R. Strand, G. Giovannetti, B. Hellsing, L. de'Medici, and M. Capone, Phys. Rev. B 87, 045122 (2013).

${ }^{19}$ K. Haule and G. Kotliar, New J. Phys. 11, 025021 (2009).

${ }^{20}$ Y. Li, Z. P. Yin, X. C. Wang, D. W. Tam, D. L. Abemathy, A. Podlesnyak, C. L. Zhang, M. Wang, L. Y. Xing, C. Q. Jin, K. Haule, G. Kotliar, T. A. Maier, and P. C. Dai, Phys. Rev. Lett. 116, 247001 (2016).

${ }^{21}$ J. Fink, S. Thirupathaiah, R. Ovsyannikov, H. A. Dür, R. Follath, Y. Huang, S. Jong, M. S. Golden, Y. Z. Zhang, H. O. Heschke, R. Valentí, C. Felser, S. D. Farahani, M. Rotter, and D. Johrendt, Phys. Rev. B 79, 155118 (2009).

${ }^{22}$ L. X. Yang, Y. Zhang, H. W. Ou, J. F. Zhao, D. W. Shen, B. Zhou, J. Wei, F. Chen, M. Xu, C. He, Y. Chen, Z. D. Wang, X. F. Wang, T. Wu, G. Xu, X. H. Chen, M. Arita, K. Shimada, M. Taniguchi, Z. Y. Lu, T. Xiang, and D. L. Feng, Phys. Rev. Lett. 102, 107002 (2009).

${ }^{23}$ M. Yi, D. H. Lu, J. H. Chu, J. G. Analytis, A. P. Sorini, A. F. Kemper, B. Moritz, S. K. Mo, R. G. Moore, M. Hashimoto, W. S. Lee, Z. Hussain, T. P. Devereaux, R. Fisher, and Z. X. Shen, Proc. Natl. Acad. Sci. U.S.A. 108, 6878 (2011).

${ }^{24}$ N. Xu, P. Richard, A. Roekeghem, P. Zhang, H. Miao, W. L. Zhang, T. Qian, M. Ferrero, A. S. Sefat, S. Biermann, and H. Ding, Phys. Rev. X 3, 011006 (2013).

${ }^{25}$ M. Aichhorn, S. Biermann, T. Miyake, A. Georges, and M. Imada, Phys. Rev. B 82, 064504 (2010).

${ }^{26}$ A. Liebsch and H. Ishida, Phys. Rev. B 82, 155106 (2010).

${ }^{27}$ Z. H. Liu, P. Richard, Y. Li, L. L. Jia, G. F. Chen, T. L. Xia, D. M. Wang, J. B. He, H. B. Yang, Z. H. Pan, T. Valla, P. D. Johnson, N. Xu, H. Ding, and S. C. Wang, Appl. Phys. Lett. 101, 202601 (2012).

${ }^{28}$ D. V. Evtushinsky, V. B. Zabolotnyy, L. Harnagea, A. N. Yaresko, S. Thirupathaiah, A. A. Kordyuk, J. Maletz, S. Aswartham, S. Wurmehl, E. Rienks, R. Follath, B. Büchner, and S. V. Borisenko, Phys. Rev. B 87, 094501 (2013).

${ }^{29}$ J. Maletz, V. B. Zabolotnyy, D. V. Evtushinsky, S. Thirupathaiah, A. U. B. Wolter, L. Harnagea, A. N. Yaresko, A. N. Vasiliev, D. A. Chareev, A. E. Böhmer, F. Hardy, T. Wolf, C. Meingast, E. D. L. Rienks, B. Büchner, and S. V. Borisenko, Phys. Rev. B 89, 220506 (2014).

${ }^{30}$ Z. H. Liu, T. K. Kim, A. Sala, H. Ogino, J. Shimoyama, B. Büchner, and S. V. Borisenko, Appl. Phys. Lett. 106, 052602 (2015).

${ }^{31}$ Z. H. Liu, Y. G. Zhao, Y. Li, L. L. Jia, Y. P. Cai, S. Zhou, T. L. Xia, B. Büchner, S. V. Borisenko, and S. C. Wang, J. Phys.: Condens. Matter 27, 295501 (2015).

${ }^{32} \mathrm{~V}$. Antonov, B. Harmon, and A. Yaresko, Electronic Structure and Magneto-Optical Properties of Solids (Kluwer Academic Publishers, London, 2004).

${ }^{33}$ D. V. Evtushinsky, A. N. Yaresko, V. B. Zabolotnyy, J. Maletz, T. K. Kim, A. A. Kordyuk, M. S. Viazovska, M. Roslova, I. Morozov, R. Beck, S. Aswartham, L. Harnagea, S. Wurmehl, H. Berger, V. A. Rogalev, V. N. Strocov, T. Wolf, N. D. Zhigadlo, B. Büchner, and S. V. Borisenko, Phys. Rev. B 96, 060501(R) (2017).

${ }^{34}$ D. V. Evtushinsky, M. Aichhorn, Y. Sassa, Z. H. Liu, J. Maletz, T. Wolf, A. N. Yaresko, S. Biermann, S. V. Borisenko, and B. Büchner, e-print arXiv: 1612.02313 .

${ }^{35}$ J. P. Hu and H. Ding, Sci. Rep. 2, 381 (2012).

${ }^{36}$ A. S. Sefat, D. J. Singh, R. Jin, M. A. McGuire, B. C. Sales, and D. Mandrus, Phys. Rev. B 79, 024512 (2009).

${ }^{37}$ K. Ahilan, T. Imai, A. S. Sefat, and F. L. Ning, Phys. Rev. B 90, 014520 (2014). 